\title{
The Effects of Magnetic Activity on Lithium-Inferred Ages of Stars
}

\author{
Aaron J. Juarez ${ }^{1,2}$, Phillip A. Cargile ${ }^{2}$, David J. James ${ }^{3}$ \\ and Keivan G. Stassun ${ }^{1,2}$ \\ ${ }^{1}$ Dept. of Physics, Fisk University, \\ Nashville, TN 37208, USA \\ ${ }^{2}$ Dept. of Physics and Astronomy, Vanderbilt University, \\ Nashville, TN 37235, USA \\ email: a.juarez@vanderbilt.edu \\ ${ }^{3}$ Cerro Tololo Inter-American Observatory, \\ Casilla 603, La Serena, Chile
}

\begin{abstract}
In this project, we investigate the effects of magnetic activity on the Lithium Depletion Boundary (LDB) to recalibrate the measured ages for star clusters, using the open cluster Blanco 1 as a pilot study. We apply the LDB technique on low-mass Pre-Main-Sequence (PMS) stars to derive an accurate age for Blanco 1, and we consider the effect of magnetic activity on this inferred age. Although observations have shown that magnetic activity directly affects stellar radius and temperature, most PMS models do not include the effects of magnetic activity on stellar properties. Since the lithium abundance of a star depends on its radius and temperature, we expect that LDB ages are affected by magnetic activity. After empirically accounting for the effects of magnetic activity, we find the age of Blanco 1 to be $\sim 100 \mathrm{Myr}$, which is $\sim 30 \mathrm{Myr}$ younger than the standard LDB age of $\sim 130 \mathrm{Myr}$.
\end{abstract}

Keywords. Magnetic activity, fundamental parameters, pre-main sequence stars, open clusters

\section{Introduction}

The Lithium Depletion Boundary (LDB) technique is currently the most accurate method in estimating the age of a stellar cluster, to an uncertainty of $\sim 10 \%$. The LDB age is robust due to its insensitivity to input physics and thus gives consistent, precise ages among different PMS models (Jeffries \& Naylor 2001). Observations have shown that magnetic activity directly affects stellar temperature and radius; however, it is not accounted for in most PMS lithium depletion models (Morales et al. 2008). We use empirical corrections from Stassun et al. (2012) to account for the effect of magnetic activity on the LDB to recalibrate the measured ages for star clusters. Correcting these benchmark LDB ages for magnetic activity will improve our overall ability to measure stellar ages by comparing them to more commonly used chronometers.

\section{Analysis}

Having doubled our sample set to nearly 50 Gemini Multi-Object Spectrograph (GMOS) spectra from the Gemini-North telescope, we sought to completely reanalyze the data in a consistent manner by developing software that automates the analysis. Our data reduction pipeline reduces GMOS spectra using standard reduction routines from the IRAF Gemini-GMOS package, such as bias removal, aperture extraction, and wavelength calibration. Our recently developed spectral analysis software determines the $\mathrm{H} \alpha$ Equivalent Width (EW), Lithium I (6707.8 A) EW, radial velocity, and spectral type of the Blanco 1 candidates. 


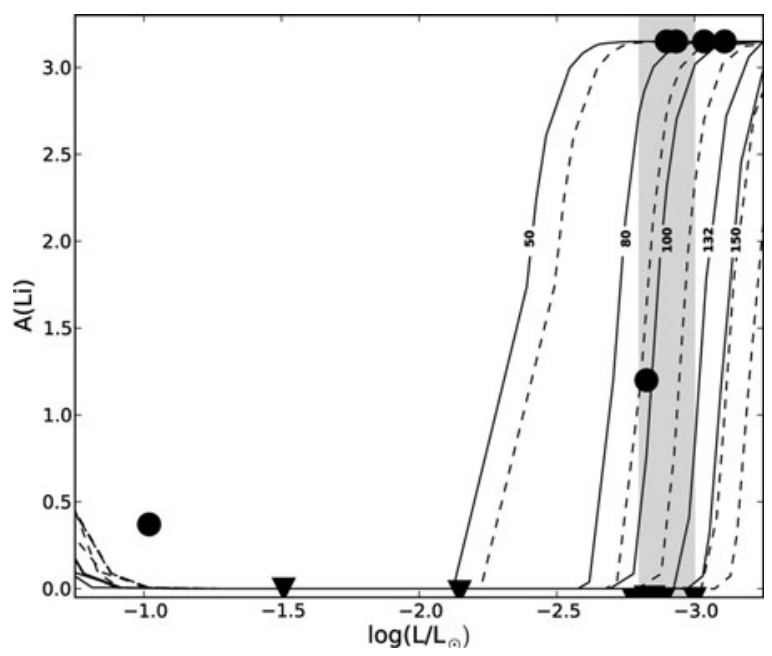

Figure 1. BCAH98 Li isochrones for various ages (lines) and our new Li abundances for Blanco 1 (points). Solid lines are the standard (non-activity) Li tracks and dashed lines are the shifted tracks which account for magnetic activity.

\section{Updated LDB Age, Empirical Correction, and Implications}

Our membership criteria was based on $\mathrm{H} \alpha$ emission, radial velocity, and proper motion of the cluster (Platais et al. 2011). Fourteen stars were considered high-confidence lowmass Blanco 1 members. The majority of these stars exhibit $\mathrm{H} \alpha$ emission as expected from chromospheric activity in young stars. To account for the magnetic activity, the BT-Settl Models of Allard et al. (2012) were used along with our measurements of $\mathrm{H} \alpha$ EW to determine changes in $T_{\text {eff }}$ and radius via the empirical relationships.

In Fig. 1, we plot new Li abundances from our sample, where circles are $>2 \sigma$ detections and triangles are $3 \sigma$ lower limits, determined from Zapatero Osorio et al. (2002). We plot BCAH98 Li isochrones for luminosity, spanning 50-150 Myr. Solid lines are standard (non-activity) Li tracks and dashed lines are shifted tracks for average changes in stellar parameters, which correspond to roughly $10 \% T_{\text {eff }}$ suppression and $10 \%$ radius inflation.

We define the LDB in Blanco 1 to be bounded by the shaded region, where we observe the $\mathrm{Li}$ abundance to go to zero. Using the BCAH98 standard predictions (solid line), we infer an updated LDB age to be near $132 \mathrm{Myr}$, which is the previously determined LDB age (Cargile et al. 2010). Including activity changes the LDB age of Blanco 1 to $\sim 100$ Myr - a difference of 30 Myr younger - indicated by the dashed line in Fig. 1. It is, therefore, worthwhile to consider the effects of magnetic activity on the inferred LDB age for other clusters with known LDBs since PMS stars often exhibit magnetic activity, which can change the timescale over which $\mathrm{Li}$ is depleted.

\section{References}

Allard, F., Homeier, D., \& Freytag, B. 2012, Phil. Trans. R. Soc. A, 370, 2765

Baraffe, I., Chabrier, G., Allard, F., \& Hauschildt, P. H. 1998, A\&SA, 337, 403

Cargile, P. A., James, D. J., \& Jeffries, R. D. 2010, ApJ (Letters), 725, 111

Jeffries, R. D. \& Naylor, T. 2011, ASPC, 243, 633

Morales, J. C., Ribas, I., \& Jordi, C. 2008, A\& $A$, 478, 507

Platais, I. Girard, T. M. and Vieira, K., Lopez, C. E., Loomis, C., McLean, B. J., Pourbaix, D., Moraux, E., Mermilliod, J.-C., James, D. J., Cargile, P. A., Barnes, S. A., \& Castillo, D. J. 2011, MNRAS, 413, 1024

Stassun, K. G., Kratter, K. M., Scholz, A., \& Dupuy, T. J. 2012, ApJ, 756, 47

Zapatero Osorio, M. R., Bejar, V. J. S.., Pavlenko, Ya., Rebolo, R., Allende Prieto, C., Martin, E. L., \& Garcia Lopez, R. J. 2002, A\&A, 384, 937 